\title{
A Erosão Sufixal dos Italianos Cultos em Contato com o Português Brasileiro (PB)
}

Tommaso Raso

RESUMO O artigo discute os mecanismos da erosão sufixal em italianos cultos em contato prolongado com o português brasileiro. $\mathrm{O}$ trabalho se baseia num corpus falado de cerca de 50.000 palavras e 13 informantes, e é homogêneo diastraticamente, diatopicamente e diafasicamente. Foram investigados os casos de perda sufixal em relação às regras de formação de palavras e à produtividade sufixal das duas línguas. As conclusões apontam para uma perda não casual mas concentrada nos sufixos menos produtivos, em particular quando a base é idêntica e a sufixação diferente, mas somente se concorre mais de um fator condicionante (tipicidade, produtividade, interferência, simplificação para uma única possibilidade a partir de mais variantes).

PALAVRAS-CHAVE erosão lingüística; sufixação; italianos no Brasil. 
ABST RACT L'articolo discute i meccanismi di erosione suffissale in italiani colti in contatto prolungato con il portoghese brasiliano. Il lavoro si basa su un corpus parlato di circa 50.000 parole e 13 intervistati, ed è omogeneo diastraticamente, diatopicamente e diafasicamente. La perdita suffissale è stata studiata in rapporto alle regole di formazione delle parole e alla produttività suffissale delle due lingue. Le conclusioni indicano una perdita non casuale ma concentrata in suffissimeno produttivi, soprattutto quando la base è identica e la suffissazione diversa, ma solo se si sommano più fattori condizionanti (tipicità, produttività, interferenza, semplificazione da più varianti a un unica possibilità).

PAROL.E CHIAVE erosione linguistica; suffissazione; italiani in Brasile.

ABSTRACT This paper discusses the mechanisms of suffix loss through attrition in the speech of educated Italians after long-drawn contact with Brazilian Portuguese Based on a spoken corpus of some 50,000 words and 13 informants, it is diastratically, diatopically and diaphasically homogeneous. The cases of suffixal loss as regards word formation rules and the suffixal productivity of both languages were investigated. The conclusions show a non casual loss affecting the least productive suffixes, particularly when the stem is identical and suffixation is different, but only if more than one conditioning factor concurs (typicality, productivity, interference, simplification to a single possibility from a number of variants).

KEYWORDS language attrition; suffixation; Italians in Brazil. 


\section{Objetivo do trabalho'}

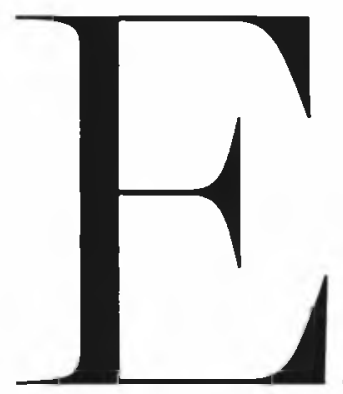

ste artigo se propõe a discutir os mecanismos da erosão sufixal (principalmente na sufixação nominal) em italianos cultos em contato prolongado com o português do Brasil. ${ }^{2}$ Com italianos cultos nos referimos a falantes italianos nativos, que viveram na Itália até à idade adulta, lá estudaram pelo menos até a conclusão do que corresponde ao Ensino Médio no Brasil, e têm formação superior, seja na Itália, no Brasil ou em outro país. Ter estudado pelo menos 13 anos na Itália ${ }^{3}$ garante que a competência lingüística deles seja sólida, e que a eventual perda lingüística não seja conseqüência de uma aquisição somente parcial; ter formação superior garante uma capacidade metalingüística que permita uma reflexão e um controle maior sobre a própria produção. Estes falantes devem estar morando no Brasil há pelo menos oito anos, tempo suficiente para terem adquirido uma competência quase nativa do PB. Todos os informantes do corpus utilizado para a presente pesquisa viveram no Brasil por mais de vinte anos. ${ }^{4}$ Com erosão entendemos, na definição de Köpke e Schmid (2004:5), "uma redução não patológica da proficiência de uma língua que fora adquirida anteriormente por um indivíduo, ou seja, uma perda intrageneracional" 5

1. Com a colaboração de Ernani Almeida.

2. O trabalho se insere num projeto de pesquisa coordenado por T. Raso e financiado por meio de bolsas Fapemig e Pro-Grad UFMG. Mais detalhes em Raso no prelo.

3. Os estudos pré-universitários na Itália são de 13 anos, já que o equivalente do segundo grau é de 5.

4. Para uma discussão sobre os fatores sociolingüísticos que afetam a análise de dados sobre erosão de Ll, veja-se Köpke; Schmid 2004:19-23, em que, entre outras coisas, aponta-se para o fato de que a erosão acontece durante a primeira década de afasta mento da comunidade de Ll. A bibliografia sobre o italiano em contato com outras línguas é muito rica, mas os trabalhos sobre a erosão do italiano estudam em grande maioria comunidades de emigrantes com baixo nivel de escolarização, nas quais não é possível separar claramente o que é devido a erosão e o que a limites na aquisição. Outros estudos analisam a erosão em falantes de segunda ou terceira geração que, apesar de ter aprendido e usar o italiano em casa, internalizaram desde o início uma língua que apresenta efeitos da erosão na geração anterior, os assim achamados de "heritage speakers"; isso põe o ulterior problema de estabelecer o que esses falantes realmente conheciam antes que o processo de erosão individual começasse (Sorace 2004:143). Entre esses estudos veja-se Jaspaert e Kroon 1988 e 1989 para italianos em contato na Holanda; para o contato nos EUA, Haller 1993, Scaglione 2000; para o contato na Austrália, Bettoni 1981,1986,1990, 1991a e 1991b; para outros países, inclusive da América Latina, vejam-se algumas das contribuições em Vanvolsem, S.-Vermandere, D.-Musarra, F.-D’Hulst, Y. 2000 e a bibliografia citada; quanto à América do sul, existe também uma rica bibliografia sobre a erosāo dos dialetos vênetos no Brasil e em outros países. Os pouquíssimos estudos sobre italianos cultos estudam realidades em contato com o inglês (Tsimpli et alii 2003 e Sorace 2000 e 2004), com a exceção de Raso 2003 que estuda a realidade brasileira, mas nenhum desses trabalhos aprofunda a erosāo sufixal.

5. "non-pathological decrease in proficiency in a language that had previously been acquired by an individual, i. e. intragenerational loss". Para uma discussão teórica sobre o conceito, veja-se também Selinger e Vago 1991; Cook 2003; Schmid et al. 2004. 
Provavelmente algumas áreas da gramática da língua podem ser comprometidas mais do que outras, incluindo no conceito de erosão tanto fenômenos de perda de regras ou de fragmentos do sistema sob a pressão da L2, quanto a simplificação do sistema por falta de insumo. ${ }^{6}$ Mas mesmo na busca dessa hierarquia deve-se lidar com várias possíveis causas, muitas vezes concorrentes, como a dominância de uma língua, a maior ou menor complexidade em relação a uma estrutura entre as línguas em competição, além da especificidade estrutural do traço em questão.?

No caso da sufixação, o uso de um sufixo diferente daquele usado pelos monolíngües pode, em princípio, ter várias explicações. A troca de sufixos pode acontecer por interferência de uma palavra, de mesmo significado ou de significante parecido, da L2; por interferência da produtividade de uma regra de formação de palavras (RFP) na L2 na produtividade da RFP correspondente da Ll; por diferença de freqüência de uso de RFPs correspondentes entre as duas línguas; por processos de simplificação em que uma RFP menos produtiva cede em confronto a RFPs mais produtivas dentro da própria Ll; por mais de um motivo ao mesmo tempo.

Raso (2003) já havia mostrado que a erosão da sufixação na LI (italiano) em contato prolongado com a L2 (PB) ocorre. Pretendemos agora investigar o fenômeno com relação a seu nivel de profundidade e descobrir algo a mais sobre as causas. Se for apenas no nível lexical, a erosão poderia ocorrer com qualquer sufixo e, portanto, não haveria motivos para um sufixo se erodir mais do que outro. Se, ao contrário, o que se perde são as regras de formação das palavras, os sufixos menos produtivos no italiano seriam afetados mais em favor dos mais produtivos no português ou no próprio italiano. Outras possibilidades chamariam em causa mais de um fator. Os resultados apresentados neste trabalho não permitem uma conclusão definitiva, devido à insuficiência do corpus, mas fornecem alguns indícios interessantes que permitem a formulação de hipóteses a serem testadas quando estiver disponível um corpus mais amplo. ${ }^{8}$

\section{A produtividade sufixal em italiano e no português brasileiro}

Quando falamos em sufixos produtivos e improdutivos, na realidade, estamos falando de regras de formação de palavras (RFP) produtivas e improdutivas. ${ }^{9}$ A produtividade não é simples de ser definida. Com o termo produtividade podem-se considerar vários modos de medir o emprego de uma RFP. Para este trabalho adotamos o seguinte conceito de produtividade: "A probabilidade de que um dado afixo ou um dado procedimento formativo possam produzir neologismos dentro de um período histórico determinado" 10

6. Veja-se Isurin 2000.

7. Muito interessante, a esse respeito, a discussão de Sorace (2004) do trabalho de Montrul (2002).

8. O projeto coordenado por Raso está aprontando um corpus maior, mais variado em termos de situações comunicativas, que permita o acesso ao som e transcrito por meio do sistema Childes-Clan.

9. Para a complexa questão da produtividade lexical e as diferentes maneiras para calculá-la, vejam-se Rainer 1993, Bauer 2001, Plag 2004, Gaeta-Ricca 2005a e 2005b, entre outros. Para uma abordagem psicolingüística da relação entre freqüência e analisabilidade das palavras, é útil a discussão de Laudanna e Burani 1999 e, em particular, a observação de que a baixa freqüência favorece a analisabilidade. Para o italiano os estudos são ainda poucos: vejam-se em particular Gaeta e Ricca 2003, 2005a e 2005b, Scalise 1994 e Thornton 2005 para um enquadramento geral. Ainda menos são os estudos sobre o PB (veja-se NEO, e, para um enquadramento geral, Rocha, 1998).

10. Lorenzetti 2002:61 (tradução minha). 
Um outro parâmetro que nos interessa nesse trabalho é o da freqüência sufixal, que pode ser entendida como o número de vezes que o falante usa palavras formadas a partir de uma determinada RFP: a token frequency refere-se ao número de vezes em que o sufixo é usado, seja com bases diferentes ou com a mesma base; a type frequency é o numero de palavras diferentes formadas com o mesmo sufixo em um dado corpus. Freqüência e produtividade estão relacionadas, pois a freqüência é intuitivamente ligada com a capacidade dinâmica de enriquecer o léxico mental de um falante. ${ }^{11}$ Nem a produtividade nem a freqüência de uma RFP podem ser medidas a partir do número de palavras que são atestadas no léxico da língua. Uma RFP pode ser muito freqüente, pouco numerosa e produtiva, ou vice-versa.

A Tabela 1 apresenta duas medidas de produtividade dos sufixos italianos. Os resultados se baseiam em um corpus textual constituido a partir de três anos (1996-1998) do cotidiano La Stampa de Turim, de cerca de 75 milhões de tokens.

A primeira avaliação de produtividade adotada é a relação $P=h / N$ calculada para diferentes afixos com igual valor de $N$ (Índice $P$ é igual à relação entre $h$, o número de hapax legomena ${ }^{12}$ formados com um determinado afixo presente no corpus, e $\mathrm{N}$, o número de tokens formados com o mesmo afixo presente no corpus inteiro). Para que essa medida seja operativamente confiável, os sufixos em questão devem ter freqüência em tokens não idênticas, mas confrontáveis, de modo que as dimensões dos corpora variáveis aos quais se faz referimento não tenham divergência excessiva. ${ }^{13} \mathrm{~A}$ Tabela 1 apresenta uma lista de valores de produtividade para os afixos de freqüência médio-alta, compatíveis com pelo menos um dos valores de $N=19.000,50.000$ e 100.000 .

A segunda avaliação da produtividade, a que mais nos interessa, é apresentada na última coluna da Tabela 1 ( $h$ em todo o corpus). O número de hapax legomena do afixo em todo o corpus indica a probabilidade de que um dado afixo ou um dado procedimento formativo possa produzir neologismos no período determinado.

As Tabelas 2 e $3^{14}$ apresentam a freqüência dos sufixos italianos no corpus acima descrito, respectivamente por número de tokens e por número de types.

Um olhar comparativo para as duas tabelas mostra que os sufixos mais importantes aparecem na parte alta de ambas as tabelas; portanto as freqüências em tokens e em types vão juntas para sufixos que nos interessam como -zione, -ità/età, -(t)ore, -mento, -ista. O discurso é diferente para outros sufixos: desses, o que nos interessa mais é -nza, mais freqüente em tokens do que em types.

Os resultados da Figura 1 apresentam a produtividade dos sufixos do português (variante brasileira) segundo o número de neologismos presentes no corpus constituído pelos jornais Folha de S. Paulo e O Globo e pelas revistas IstoÉ e Veja a partir de janeiro de 93, coletado segundo um sistema de amostragem (um veículo por semana). A Base conta com mais de 13.000 unidades lexicais neológicas.

11. Gaeta e Ricca 2005a:107-8.

12. Ou seja, as palavras que aparecem uma única vez no corpus.

13. Para uma discussão sobre as vantagens e os limites desse sistema de cálculo, vejam-se Baayen 1991, 1992; Baayen e Lieber 1991; Baayen e Renouf 1996; Plag et al. 1999. Para as vantagens e os limites do corpus escolhido, vejam-se Gaeta e Ricca 2003, 2005a e 2005b. Sobre a relação entre hapax legomena e neologismos, vejam-se também Gaeta e Ricca 2002: 227-229.

14. As Tabelas 2 e 3 são apresentadas com alguns ajustamentos devidos aos objetivos desse trabalho. 
Tabela $1^{15}$

\section{Alguns afixos derivacionais do italiano ordenados segundo duas medidas de produtividade}

\begin{tabular}{|c|c|c|c|c|}
\hline Afixos & $N=19.000$ & $\begin{array}{l}P(N) \times 10^{3} \\
N=50.000\end{array}$ & $N=100.000$ & $\begin{array}{c}h \text { em todo o } \\
\text { corpus }\end{array}$ \\
\hline -issimo & 25,8 & 12,9 & - & 643 \\
\hline -iano & 24,3 & - & - & 615 \\
\hline -mente & - & 10,1 & 6,4 & 825 \\
\hline -ismo & 15,2 & 8,2 & - & 448 \\
\hline -bile & 11,3 & 6,3 & 4,1 & 409 \\
\hline -ità/-età & - & 6,3 & 3,7 & 544 \\
\hline -ista & 11,3 & 6,2 & $\mathbf{3 , 8}$ & 470 \\
\hline -trice & 10,8 & & - & 224 \\
\hline -(t)ore & - & 5,0 & 3,2 & 461 \\
\hline -mento & & 4,9 & 3,1 & 402 \\
\hline -(z)ione & & - & 2,7 & 486 \\
\hline -izzare & 7,6 & 3,8 & - & 280 \\
\hline -ese & - & 3,6 & 2,2 & 244 \\
\hline$-(t) u r a$ & 6,6 & 3,5 & - & 189 \\
\hline -ale/-are & - & - & 1,9 & 155 \\
\hline -eggiare & 4,1 & - & - & 93 \\
\hline -oso & 3,7 & 1,6 & 1,0 & 127 \\
\hline$-e z z a$ & 2,7 & 1,3 & - & 70 \\
\hline -aggio & 1,5 & - & - & 29 \\
\hline$-n z a$ & 0,7 & $\mathbf{0 , 3}$ & 0,2 & 29 \\
\hline -ificare & 0,6 & - & - & 20 \\
\hline -ore & 0,4 & 0,2 & & 9 \\
\hline -evole & 0,3 & - & & 6 \\
\hline$-i z i a$ & 0,0 & - & - & $\mathbf{0}$ \\
\hline
\end{tabular}


Tabela 2

\begin{tabular}{|c|c|}
\hline \multicolumn{2}{|c|}{$\begin{array}{l}\text { Sufixos derivacionais italianos ordenados por classe logarítmica de freqüência } \\
\text { (em tokens) }{ }^{16}\end{array}$} \\
\hline Sufixos ${ }^{17}$ & Classe (in $N$ ) \\
\hline$-(z)$ ione & 14 \\
\hline -ale/-are, -ità/-età,-mente, -(t)ore & 13 \\
\hline -mento, -nza, -ista, -oso, -ese, -bile & 12 \\
\hline -izzare, -ore, -ezza, -(t)ura, -ismo, -iere, -issimo, -izia, -iano & 11 \\
\hline -ificare, -eggiare, -trice, -aggio, -evole, -eria ${ }^{(2)}$, -iero, -(t)orio & 10 \\
\hline -aio ${ }^{(a)}$, -iera, -esco, super-, -essa, -ificio, -toio/a, -izio & 9 \\
\hline -accio, -aneo, -ame, -erì ${ }^{(1)}$, -aio/a ${ }^{(2)}$, micro-, mini- & 8 \\
\hline iper-, maxi-, -estre, ultra-, mega-, -aglia, -oide, -aggine, -ume, & 7 \\
\hline -astro, -eto/a & 6 \\
\hline -aceo, -igia & \\
\hline
\end{tabular}

\section{Tabela 3}

Sufixos derivacionais italianos ordenados por classe logarítmica de numerosidade ${ }^{18}$

\begin{tabular}{|c|c|}
\hline Sufixos & Classe (in V) \\
\hline -mente, -(z)ione, -ità/-età & 8 \\
\hline -issimo, -ista, -(t)ore, -iano, -mento, -ismo,-bile, -ale/-are, & 7 \\
\hline -ese, -trice, -oso, -(t)ura, -esco, -ezza, -(t)orio & 6 \\
\hline -nza, -iere, -ería $a^{(2)}$, -ería ${ }^{(1)}$, -aio(1), -aggio, -iera, -oide & 5 \\
\hline $\begin{array}{l}\text {-iero, -ificio, -aggine, -aio/a }{ }^{(2)}, \text {-essa, -evole, -ore, -toio } / a, \\
\text {-aceo, -ume, -ame, -astro, -eto/a, -aglia }\end{array}$ & 4 \\
\hline -izio, -izia, -aneo & 3 \\
\hline -estre -igia & 2 \\
\hline
\end{tabular}

16. O afixo -zione apresenta no corpus mais de um milhão de ocorrências, enquanto o segundo, -ale/-are, desce imediatamente para 734.000. Em geral a classe 13 apresenta freqüências muito parecidas, enquanto as últimas 4 classes são muito pouco freqüentes (entre algumas centenas e poucos milheres).

17. - ería ${ }^{(1)}$ forma nomes de qualidades como vigliaccheria e -eria ${ }^{(2)}$ nomes de lugar como libreria; -aio ${ }^{(1)}$, nomes de agente como fioraio e -aio(2) nome de lugar como pollaio.

18. O corpus vai dos 2.767 types de -mente e os 2.363 de -zione até os 7 dos dois menos freqüentes. 


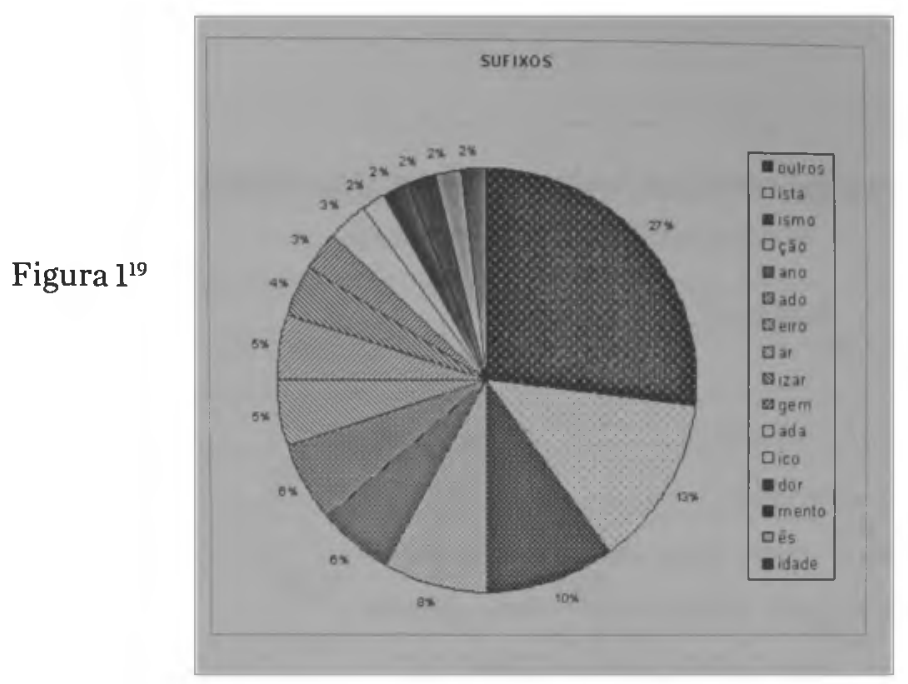

\section{Metodologia}

A partir da análise da produtividade e da freqüência dos principais sufixos, individuamos alguns pares correspondentes em italiano e português para uma análise mais aprofundada. Escolhemos em princípio pares de sufixos com baixa, média e alta produtividade em ambas as línguas. Em seguida analisamos os casos de erosão relativos a todos os sufixos que aparecem no corpus.

A primeira análise foi realizada sobre oito pares de sufixos. Seis dos pares de sufixos apresentam produtividade semelhante: com baixa produtividade foram escolhidos os sufixos-aggio/-gem;-ura/ -ura e-iere, -aio, -aro, -ario/-eiro, -ario; ${ }^{20} \mathrm{com}$ média produtividade o sufixo-mento/-mento; e com alta produtividade os sufixos -ista/-ista e-zione, -sione/-ção, -são. ${ }^{21}$ Os outros dois pares desufixos apresentam produtividade razoavelmente diferente nas duas línguas: o sufixo -ità, -età é muito produtivo em italiano, mas seu correspondente português, -idade, é pouco produtivo; e o par -bile, -vole/-vel ${ }^{22}$ tem o sufixo italiano bem acima na escala de produtividade de quanto esteja o sufixo português. Cabe ressaltar que entre os sufixos escolhidos temos dois pares de sufixos concorrentes: ${ }^{23}$ -ista/-eiro, -ario (port.); -ista/-iere, -aio, -aro, -ario (it.) e -ção/-mento (port.); -zione/-mento (it.). A concorrência entre os sufixos -ista e-eiro (-ista e -iere, -aio, -aro em italiano) não acontece da mesma forma como entre -ção e -mento (-zione e-mento). Embora os sufixos -ista e -eiro (assim como os correspondentes italianos) formem substantivos agentivos a partir de substantivos, temse observado que eles obedecem atualmente a um paradigma. O sufixo -eiro (e o correspondente

19. Fonte da figura l é NEO: http://www.fflch.usp.br/dlcv/neo/Dados_quantitativos.htm. Dados quantitativos. Acessado pela última vez em: $29 / 10 / 2007$.

20. O sufixo agentivo do português, -eiro, corresponde aos três sufixos do italiano-iere, -aio,-aro: -aro è uma variante de -aio que se difundiu por meio de palavras de origem diatópica diferente daquela de-aio mas com a mesma função. Enquanto-aio è de origem toscana, e portanto desde sempre italiana, - aro è originário de muitas outras partes da Itália, especialmente Roma e Nápoles, e se difundiu a partir da entrada de muitas palavras não toscanas no léxico do italiano. Quanto a-iere, sua origem é francesa, mas essa forma está agora em produtividade muito regressiva. A última variante, -ario, é o sufixo de transmissão culta, próprio do latim.

21. A rigor, os sufixos -zione/-ção e -sione/-são devem ser vistos como sufixos diferentes: o primero se aplica ao tema do infinitivo, enquanto o segundo ao tema do participio (Marini, 2003:21).

22. Os dois sufixos italianos correspondem ao mesmo sufixo do português.

23. "Sufixos concorrentes são aqueles que, embora distintos sob o ponto de vista fonético, apresentam o mesmo sentido e/ou funçăo [...]. Para serem concorrentes, as bases e os produtos precisam pertencer à mesma categoria lexical" (Rocha, 1998:112). 
italiano -aio) hoje é produtivo, sobretudo, para designar profissões ligadas a atividades manuais, braçais ou com conotação negativa. ${ }^{24} \mathrm{O}$ sufixo -ista, ao contrário, forma neologismos relacionados a atividades intelectuais. Esse paradigma é percebido intuitivamente pelos falantes nativos.

Para a análise se usou o corpus que foi publicado na Revista de Italianística. Esse corpus consiste em doze entrevistas e uma conversação livre realizadas entre 1994 e 1996. Dez das entrevistas são face a face e duas são telefônicas. O total de palavras, contando exclusivamente as dos informantes, é de cerca de 50.000 .

Para o sufixo -zione/-ção, foram contados 72 types e 184 tokens; 3 types ( 4 tokens) apresentam erosão sufixal, ou seja, o sufixo padrão do italiano é outro. É necessário dizer que em todos os outros casos o sufixo do italiano e do correspondente português é igual, o que reduz fortemente a probabilidade de erosão. Únicas exceções são o caso de consultazione>consulta e o caso de registrazione/registro. Para o sufixo -sione/-são foram contados 12 types e 28 tokens, com uma correspondência total entre a sufixação italiana e a do português.

Faz-se necessário também considerar que não todos os itens sufixados são percebidos da mesma maneira pelo falante: do ponto de vista sincrônico, algumas palavras são ainda segmentáveis; outras, como por exemplo delusione, diffusione, previsione, iscrizione, persecuzione, formazione e outros provavelmente não podem ser considerados sufixados da mesma maneira que itens como fondazione (que tem um correspondente fondamento e que poderia ter correspondentes como *fondatura ou *fondaggio) e outros, cuja segmentabilidade é claramente percebida pelos falantes. Não se dá aqui uma diferenciação entre types segmentáveis e não segmentáveis, pois se trata de uma questão de continuum e de intensidade, mas essa consideração deve ser estendida a todos os sufixos; a seleção devida à não segmentabilidade, combinada com o fato de que na grande maioria dos casos os itens italianos e portugueses correspondentes apresentam a mesma base e o mesmo sufixo, implica que um trabalho definitivo sobre a erosão sufixal precisaria de um corpus muito maior que o corpus sobre o qual se trabalhou e que, no momento, é o único disponivel.

Para o sufixo -ità/-età foram contados 51 types e 171 tokens, com um único caso de erosão. ${ }^{25}$ Nesse sufixo a correspondência entre italiano e português se dá em todos os casos menos dois. Para o sufixo - mento/-mento temos 35 types e 84 tokens, com 15 itens que não apresentam correspondência: em 12 casos a falta de correspondência envolve somente o sufixo (apesar de que em alguns casos talvez fosse preferivel também uma mudança de base); 3 casos não apresentam em português a mesma base do italiano. Além disso aparecem 2 casos de erosão em que a sufixação padrão do italiano não prevê - mento, ${ }^{26} \mathrm{e}$ em 1 dos casos - mento não pertence nem ao italiano padrão nem ao português. ${ }^{27}$ Para os sufixos -aio, -aro,-iere, -ario/-eiro, -ario temos apenas 12 types e 27 tokens (sendo que 10 são do item letterario). Aqui a correspondência entre Ll e L2 é total (não sempre com a mesma variante sufixal) menos 1 , e os casos de erosão são $2{ }^{28}$ Para o sufixo -ista/ -ista temos 11 types e 20 tokens. Um único type não apresenta correspondente sufixal nas duas línguas. O único caso de erosão é relativo à base (motorista em italiano padrão é autista), e portanto

24. Lorenzetti 2002: 61-62 para o italiano e Alvares, sem data, para o português do Brasil. 25. ansietà, em que prevalece o sufixo do português ansiedade sobre o do italiano ansia.

26. relazionamento, com duas ocorrências, onde prevalece o sufixo do português relacionamento, enquanto o italiano relazione não é mais segmentável.

27. ostacolamento, onde ambas as línguas não possuem a sufixação -mento: ostacolo/obstáculo.

28. Scritturaio, em que a in terferência do português escriturário parece substituir o italiano scrivano, e immobiliaria, onde a sufixação do português prevalece sobre o italiano immobiliare. 
foge do escopo desse trabalho. O sufixo -bile, -vole/-vel aparece em 10 types e 18 tokens, com um único caso de falta de correspondência e nenhum caso de erosão. O sufixo-ura/-ura aparece em 8 types e 18 tokens com 2 casos de falta de correspondência e nenhum de erosão. O último sufixo, -aggio/-agem, aparece em apenas 2 types e 4 tokens, sempre correspondentes nas duas línguas.

O corpus portanto apresenta uma token frequency dos sufixos analisados, segundo a ordem seguinte: -zione; -ità/-età; -mento; -sione; -aio/-aio/-iere/-ario; -ista; -bile/-vole; -ura; -aggio. Essa seqüência respeita aquela das tabelas 2 e 3 , com a exceção de -ista, que aparece mais embaixo do que o normal na lista de freqüência. Em se tratando de um corpus tão limitado em relação ao fenômeno analisado, o dado não tem valor confiável algum. Mas é interessante notar que o impulso à produtividade do sufixo -ista é muito recente, ${ }^{29}$ e que todos os informantes são pessoas que, já na metade dos anos noventa, viviam fora da Itália há pelo menos 20 anos, e muitas vezes há mais de 40 .

Os casos de sufixação nominal não padrão encontrados são registrados na tabela 4, na qual se inserem também os itens que não pertencem aos sufixos inicialmente pesquisados. Dada a escassa documentação sobre o fenômeno dentro de um corpus de 50.000 palavras, resolvemos olhar para todos os casos de erosão na sufixação nominal para tentar uma primeira explicação.

Tabela $4^{30}$

\begin{tabular}{|l|l|l|l|c|}
\hline \multicolumn{1}{|c|}{ Types não padrão } & \multicolumn{1}{|c|}{$\begin{array}{c}\text { Correspondentes } \\
\text { em PB }\end{array}$} & Forma padrão & tokens \\
\hline 1 & Adattazione (II,54) (II,142) & Adaptação & Adattamento & 2 \\
\hline 2 & Orientazione (I,730) & Orientação & Orientamento & 1 \\
\hline 3 & Provazione (VII,41) & Provação & Prova & 1 \\
\hline 4 & Relazionamento(IV,19) (IV,77) & Relacionamento & Relazione & 2 \\
\hline 5 & Ostacolamento (V,458) & Obstáculo & Ostacolo & 1 \\
\hline 6 & Ansietà (VII,117) & Ansiedade & Ansia & 1 \\
\hline 7 & Assassinati (VII,574) & Assassinatos & Assassinii & 1 \\
\hline 8 & Viaggiante (II,363) & Viajante & Viaggiatore & 1 \\
\hline 9 & Scolarità (I,41) & Escolaridade & Scolarizzazione & 1 \\
\hline 10 & Scritturaio (IV,42) & Escriturário & Scrivano & 1 \\
\hline 11 & Immobiliaria (VIII,281) & Imobiliária & Immobiliare & 1 \\
\hline 12 & Ormonio (XII,84) & Hormônio & Ormone & 1 \\
\hline 13 & Consulta (XIII,36) & Consulta & Consulto & 1 \\
\hline 14 & Consulta (VIII,465) (VIII,466) & Consulta & Consultazione & $\mathbf{2}$ \\
\hline 15 & Riferenza (VII,800) & Referência & Riferimento & $\mathbf{1}$ \\
\hline 16 & Fisioterapeuta (IX,272) & Fisioterapeuta & Fisioterapista & $\mathbf{1}$ \\
\hline & & & & 19 \\
\hline
\end{tabular}

29. Lorenzetti 2002: 62. Para uma análise detalhada sobre os neologismos italianos do séc. XX, vejam-se Thornton; Iacobini 1992 el997; Thornton; Iacobini; Burani, 1997.

30. Indicamos com número romano o inquérito e com número árabe a linha. 
A Tabela 3 mostra os casos de sufixação nominal; à margem do estudo, podemos acrescentar três casos não padrão de sufixação adjetival, dois de sufixação verbal e um adverbial:

1) culto spirita (IV, 277) < spiritista;

2) vocabolario basico (VIII,421) < vocabolario di base;

3) folclorici $(1,1308-9)<$ folcloristici;

4) espressarsi (VII,692) < esprimersi;

5) residito (IX, 18) < risieduto;

6) gradativamente (VII,121) < gradatamente.

\section{A análise}

Analisando os dados, notamos as seguintes mudanças, quanto à sufixação nominal:

1) os casos de sufixação registrados na tabela como não padrão são um número limitado: 19 tokens e apenas 15 types, dois dos quais (consulta) correspondem a duas diferentes sufixações em italiano;

2) sufixos concorrentes e mais freqüentes (como -zione e -mento, mas não só) são envolvidos mais no processo de erosão;

3) 3 casos (2 types) são de -mento>-zione (os pontos 1 e 2 da tabela), e portanto explicáveis com a preferência por um sufixo concorrente mais produtivo em ambas as línguas; um desses casos, porém, aparece em um contexto de autocorreção (adattazio-adattarsi), em que o falante muda a estratégia, passando da nominal à verbal, mostrando talvez não possuir, pelo menos no momento, a sufixação certa, mas também mostrando uma percepção do problema;

4) 4 casos (3 types) são de $\varnothing$ >-mento (os pontos 4-5 da tabela), também explicáveis com o fato de o sufixo $\emptyset$ ser pouquíssimo produtivo em italiano e em português. No italiano, ele resiste somente em âmbito burocrático e político. ${ }^{31} \mathrm{O}$ caso de relazione não pode ser considerado dentro dos sufixados em -zione, pois é inanalisável pelos falantes, constituindo um sufixado do latime não do italiano. ${ }^{32}$ Valea pena aqui anotar um caso de erosão não registrado na tabela: incamminamento (VII,508); o caso não foi registrado na tabela porque parece mais um caso de erosão da base: o padrão seria avviamento, mas alguns dicionários registram até a possibilidade incamminamento; ${ }^{33}$ de toda maneira, o italiano 
possui com freqüência as bases cammino e incamminarsi. Tudo isso gera uma pressão para a erosão que se soma à opção da L2. Um dos casos (ostacolo>ostacolamento) é especialmente interessante, pois mostra que a tendência não precisa necessariamente se apoiar na forma da $L 2$.

5) 1 caso é de $\varnothing$ >-età (o ponto 6 da tabela), também explicável como preferência por um sufixo muito mais produtivo nas duas línguas. No entanto esse caso merece algum comentário: ansietà existe no léxico italiano, mas é muito menos usado do que o quase sinônimo ansia e principalmente pouco apropriado no contexto;

6) 1 caso é de $\emptyset>-a t o$, ou seja, de passagem de um sufixo quase completamente improdutivo para um sufixo bem mais produtivo: o sufixo - ato não aparece no elenco dos sufixos produtivos já que, enquanto sufixo que forma o particípio passado, é um sufixo gramatical e não somente derivacional; isso gera dificuldades na identificação da natureza nominal ou verbal das ocorrências, e portanto é excluído da pesquisa que baseia as Tabelas 1 e 2. Dada essa premissa, esse caso também se explica com a preferência por um sufixo mais produtivo;

7) os 6 casos de $\varnothing>$ sufixo pleno mostram a necessidade de marcar claramente a categoria nominal, que na sufixação zero não é transparente. Esse é um fenômeno que reflete o enfraquecimento das noções categoriais morfossintáticas em ausência de um marcador explícito, e chama a atenção para o princípio da tipicidade como referência para o falante submetido à erosão, de uma forma parecida com o que acontece com o falante em fase de aquisição. ${ }^{34}$ Trata-se de uma consideração extremamente interessante se considerarmos a hipótese jakobsoniana da regressão, ${ }^{35}$ segundo a qual o que se aprende depois é também o que se perde primeiro; ${ }^{36}$ segundo esse princípio, a erosão lingüística deveria olhar não somente para as L2 de contato, mas também para as seqüências aquisicionais da $\mathrm{Ll}$;

8) o caso de viaggiatore >viaggiante muda um sufixo pouco produtivo e bastante freqüente do italiano para um outro sufixo produtivo e freqüente, mas não considerado na pesquisa sobre a produtividade enquanto ao mesmo tempo sufixo derivacional e flexional: -nte serve também para formar particípios presentes e se torna difícil definir o valor verbal, adjetival ou nominal de cada item; vale a pena ressal tar que em italiano hoje o sufixo é produtivo apenas como sufixo nominal e adjetival, e não mais como sufixo verbal, com a exceção de poucos termos e unicamente no domínio burocrático. ${ }^{37}$ É compreensível, portanto, que nesse quadro a convergência com o sufixo do português seja mais provável;

9) 1 caso é de -izzazione >-ità, mudando de um sufixo muito produtivo para outro também muito produtivo. Aqui é necessário considerar que: em primeiro lugar o italiano possui tanto

34. A ampla produção sobre aquisição, tanto de Ll quanto de L2, chamou a atenção sobre a questão da tipicidade como princípio guia na marcação de categorias morfológícas em estágios de aprendizagem intermediários.

35. Jakobson 1971.

36. Para um trabalho de testagem desse princípio, veja-se Keijzer, 2004.

37. Raso 2005: 116. 
scolarizzazione quanto scolarità, mas com significados ligeiramente diferentes, cuja definição não é fácil, apesar de um falante nativo perceber os diferentes contextos de uso. ${ }^{38}$ Isso gera uma forte pressão para a simplificação, que aumenta considerada a pressão da L2; em segundo lugar, o sufixo abandonado é na realidade um duplo sufixo, o que pode talvez aumentar a pressão para a simplificação; ${ }^{39}$

10) quanto ao caso do ponto 10 , precisamos de mais cuidados: scritturaio não se limita a mudar o sufixo do italiano scrivano, mas muda também o radical (o que poderia deixar esse caso fora do escopo do trabalho); isso pode ser explicado de duas maneiras: por um lado como simples substituição lexical (a erosão seria portanto exclusivamente em nível lexical), por outro considerando que o tema scrit- é presente no italiano scrittore, e em outros itens lexicais da mesma área semântica como scrittura, bem mais freqüentes do que scrivano ou scrivente com o tema scriv-. Não se pode portanto excluir que a erosão aqui opere tanto no nível do sufixo quanto no nível da base, preferindo em ambos um concorrente mais freqüente;

11) l caso é de -mento>-nza (número 15 na tabela: riferimento > riferenza), ou seja, a passagem de um sufixo mediamente produtivo a um outro com o mesmo nível de produtividade em italiano; em português - mento é bem menos produtivo do que em italiano, mas o sufixo-nça nem aparece no gráfico 3. Mas o italiano oferece também o termo referenza, mesmo se com outro sentido. $\mathrm{O}$ caso seria compatível com uma explicação simplificatória que reduziria à unidade uma pluralidade sufixal da Ll em uma situação em que a L2 permite uma única possibilidade;

12) o caso de consulto >consulta parece mais uma mudança de gênero do que de sufixo, e portanto não interfere no quadro;

13) o caso de consultazione >consulta (2 ocorrências) parece exatamente o oposto do caso apresentado nos números 3 e 4 dessa lista, com a diferença que o sufixo em questão é -zione, um sufixo altamente produtivo e do qual não se esperaria a perda. Todavia, como acontece também num caso de -mento >-zione (veja ponto 2 da lista), aqui também temos autocorreção numa das duas ocorrências, e dessa vez a autocorreção é completa: consulta-consultazione; além disso, o italiano também possui a palavra consulta, mesmo que em outro sentido, o que permitiria uma explicação parecida com o caso 11;

14) o caso de ormone>ormonio, com duas diferentes terminações a partir do anglicismo (de derivação grega) hormon, ${ }^{40}$ não traz nenhuma dificuldade, já que a terminação -io em italiano é muito freqüente;

38. Segundo o DISC, scolarità é "o dado estatístico da freqüência escolar", enquanto scolarizzazione é "diffusione e controllo dell'obbligo scolastico". O contexto de uso do nosso caso prevê scolarizzazione.

39. A práxis nos cálculos sufixais é considerar somente os sufixos externos (Plag et al. 1999: 29), mas os casos de sufixação múltipla constituem um problema, e os sufixos internos podem às vezes ter impacto, como acontece, quanto ao italiano, para o sufixo -izzare, entre outros (veja-se Gaeta e Ricca 2003, 2005b).

40. DELI, s. v. 
15) o caso de immobiliare >immobiliaria se explica com o fato de que o italiano possui ambos os sufixos, como o português, mas que no sufixo -are o equivalente português (-ar) é fonologicamente incompativel com o italiano, que exige a terminação vocálica; além disso, os dois sufixos são foneticamente muito parecidos, o que ajuda a explicar a troca;

16) o último caso, fisioterapista $>$ fisioterapeuta, se explica com a seguinte situação: em italiano existem tanto terapeuta quanto terapista; o primeiro é muito mais frequente do que o segundo, que aparece mais somente quando é o segundo membro de um composto.

\section{Conclusões}

Se essa análise é correta, não podemos não notar que a erosão sufixal não é um fenômeno casual. O que parece ter maior interesse é que, em todos os casos, mais de um motivo concorre para explicar a erosão: a maior produtividade, aliada à interferência da L2 e da L1, bem como à tipicidade, concorre para explicar o caso de ansietà; a produtividade mais a interferência da L2 mais a tipicidade concorrem para explicar os casos de provazione, relazionamento, assassinati, viaggiante, scritturaio; a interferência, tanto da L2 quanto da Ll, mais a produtividade concorrem para explicar o caso de fisioterapeuta; produtividade e interferência da L2 concorrem para explicar adattazione, orientazione, immobiliaria, ormonio; interferências da L2 e da Ll concorrem para explicar consulta e riferenza; produtividade e tipicidade concorrem em explicar ostacolamento. Esse quadro, portanto, nos permitiria avançar a seguinte hipótese: a interferência da L2 é sem dúvida um fator importante de erosão e de fato aparece em 14 dos 15 types individualizados; mas não pode ser considerada um fator único, nem um fator suficiente, pois é sempre acompanhado por, pelo menos, um outro fator. Em 10 casos concorre o fator produtividade, em 6 o fator tipicidade e em 5 a interferência da própria Ll. A importância desses outros fatores não é apenas testemunhada pela freqüência relativa com que concorrem a gerar erosão, mas também pelo fato de que, em um caso (ostacolamento), a interferência da L2 nem participa, e tipicidade e produtividade juntas demonstram ter a capacidade de gerar erosão sem que a L2 ofereça uma forma de apoio.

O presente trabalho, portanto, por um lado mostra como o fenômeno da erosão da sufixação nominal precisa de um corpus maior, suficiente para a individualização de um número de ocorrências suscetível de tratamento estatístico; por outro lado permite formular a hipótese de que o fenômeno não se gera de maneira casual, mas pela pressão de mais forças, das quais nenhuma parece ser nem suficiente nem necessária para a emergência do fenômeno. 


\section{Bibliografia}

ALVARES, C. A. Sufixos formadores de profissão em português: -ISTAX-EIRO. Uma oposição. www.filologia.org.br/ viicnlf/anais/caderno04-14.html, sem data.

BAAYEN, H. Quantitative aspects of morphological productivity. Yearbook of

Morphology, 1991:109-149.

. On frequency, transparency and productivity. Yearbook of Morphology, 1992:181-208.

BAAYEN, H.; LIEBER, R. Productivity and English word-formations: a corpus-based study. Linguistics 29, 1991:80143.

BAAYEN, H.; RENOUF, A. Chronicling the Times: productive lexical innovations in an English newspaper. Language 72, 1996:69-96.

BAUER, L. Morphological Productivity. Cambridge: Cambridge University Press, 2001.

BETTONI, C. Italian in North Queensland. Changes in the speech of first and second generation bilinguals. Townsville: James Cook University of North Queensland, 1981.

. Italian language attrition in Sidney: the role of birth order. In: BETTONI, C.; DE MAURO, T.; CRINO, P.; KINDER, J. J. (Orgs.) Italian Abroad: Studies on language contact in English-speaking countries. Sidney: Frederick May Fndtn. for Italian Studies, 1986:61-85.

. Italian language attrition in Sydney: The role of dialect. In: HALLIDAY, M.A.K. et al. (Orgs.). Learning, keeping, and using language, Vol. II. Amsterdam: John Benjamins, 1990:75-89.

Language variety among Italians: anglicisation, attrition, attitudes. Em: Romaine, S. (Org.). Language in Australia. Cambridge: Cambridge University Press, 1991:263-269.

. Language shift and morphological attrition among second generation Italo-Australians. Rivista di linguistica, 3, 1991:369-387.

COOK, V. (Org.), Effects of the second language on the first. Clevedon: Multilingual Matters, 2003.

CORBIN, D. Morphologie dérivationelle et structuration du léxique. Tübingen: Niemeyer, 1987.

DELI. Dizionario etimologico della lingua italiana. Bologna: Zanichelli, 1980.

DISC. Dizionario Italiano Sabatini Coletti. Roma: Giunti, 1996.

GAETA, L.; RICCA, D. (2002). Corpora testuali e produttività morfologica: i nomi d'azione italiani in due annate della Stampa. In: BAUER, R. et al. (Orgs.) Parallela IX: "Testo - variazione - informatica / Text - Variation - Informatik" (Salzburg 1-4/11/2000), Wilhelmsfeld: Egert, 2002:223-249.

Frequency and productivity in Italian derivation: a comparison between corpus-based and lexicographical data. In: ARONOFF, M.; GAETA, L. (Orgs.). Special Issue on Morphological Productivity. Italian Journal of Linguistics; Rivista di Linguistica, 15, 2003:63-98.

Aspetti quantitativi della produttività morfologica. In: DE MAURO, T.; CHIARI, I. (Orgs.). Parole e numeri: analisi quantitative dei fatti di lingua. Roma: Aracne, 2005a:107-124.

. Davide, Productivity in Italian morphology: a variable-corpus approach. Linguistics, 2005 b.

Productivity in Italian word formation: a variable-corpus approach. Linguistics: an interdisciplinary journal of the language sciences, 44, 1, 2006:33-57.

GARZANTI. Grande Dizionario d'Italiano. Milano: Garzanti, 2008.

HALLER, H. Una lingua perduta e ritrovata. Firenze: La Nuova Italia, 1993.

IACOBINI, C.; THORNTON, A. M. Tendenza nella formazione delle parole nell'italiano del ventesimo secolo. In: MORETTI, B.; PETRINI, D.; BIANCONI, S. (Orgs.) Linee di tendenza dell'italiano contemporaneo., Bulzoni, Roma: 1992:22-55.

ISURIN, L. “Deserted Island” or a child's first language forgetting. Bilingualism: Language and Cognition 3(2), 2000:151-166. 
JAKOBSON, R. Il farsi e il disfarsi del linguaggio (linguaggio infantile e afasia). Torino: Einaudi, 1971 (1941).

JASPAERT, K.; KROON, S. The relationship between language attitudes and language choice. In: VAN HOUT, R.; KNOPS, U. (Orgs.) Language Attitudes in the Duch Language Area. Dordrecht: Foris, 1988:157-172. . Social determinants of language loss. ITL Review, 83-84, 1989:75-98.

KEIJZER, M.C.J. First language attrition: a cross-linguistic investigation of Jakobson's regression hypothesis. International Journal of Bilingualism, 8.3, 2004:389-393.

KÖPKE, B.; SCHMID, M. S. Language attrition: the next phase. In: SCHMID et alii, 2004:1-42.

LAUDANNA, A.; BURANI, C. I processi lessicali; come è rappresentata la struttura morfologica delle parole? In: BENINCÁ, P.; MIONI, A.; VANELLI, L. Fonologia e morfologia dell'italiano e dei dialetti d'Italia. Atti del XXXI Congresso della Società di Linguistica Italiana, Padova, 25 settembre 1997, Roma: Bulzoni, 1999.

LORENZETTI, L. L'italiano contemporaneo. Roma: Carocci, 2002.

MARINI, E. I processi morfologici di formazione di parola dell'italiano: vicende storiche e varietà sincroniche. Em: www.italicon.it. Pisa: ICoN, 2003.

MONTRUL, S. Incomplete acquisition and attrition of Spanish tense/aspect distinctions in adult bilinguals. Bilingualism: Language and Cognition, 5, 2002:327-340.

NEO. Observatório de neologísmos científicos e técnicos do português contemporâneo do Brasil. http://www.flch. usp.br/dlcv/neo/Dados_quantitativos.htm

PLAG, I. Productivity. Encyclopedia of Language and Linguistics $2^{\text {nd }}$ ed. Elsevier, 2004.

PLAG et al. Morphological productivity across speech and writing. English Language and Linguistics, 3, 1999:209228.

RAINER, F. Spanische Wortbildungslehre. Tübingen: Niemeyer, 1993.

RASO T. L'italiano parlato a S. Paolo da madrelingua colti: primi sondaggi e ipotesi di lavoro. Revista de Italianística, 8, 2003:9-49.

. La scrittura burocratica. Roma: Carocci, 2005.

. L'erosione linguistica dei madrelingua italiani colti in contatto prolungato col portoghese brasiliano: un progetto in corso. Atas do XI Congresso Nacional da ABPI I IV Encontro Internacional de Estudos Italianos e I Jornada de Italianistica do Mercosul (Foz do Iguaçu, 2005) (no prelo).

Revista de Italianistica, 5, 1997, pp. 29-272.

ROCHA, L. C. A. Estruturas Morfológicas do Português. Belo Horizonte: Ed. da UFMG, Coleção Aprender, 1998.

SCAGLIONE, S. Attrition. Milano: Francoangeli, 2000.

SCALISE, S. Morfologia. Bologna: Il Mulino, 1994.

SCHMID, M. S. et al. First Language Attrition: Interdisciplinary Perspectives on Methodological Issues. Cambridge: Cambridge University Press, 2004.

SELINGER, H. W.; VAGO, R. M. (Orgs.) First Language Attrition. Cambridge: Cambridge University Press, 1991. SERIANNI, L. Italiani scritti. Bologna: Il Mulino, 2002.

SORACE, A. Differential effects of attrition in the L1 syntax of near-native L2 speakers. In: HOWEL, S. C.; FISH, S. A.; KEITHH-LUCAS, T. (Eds). Proceedings of the $24^{\text {th }}$ Annual Boston University Conference on Language Development. Somerville: Cascadilla Press, $2000: 719-725$.

. Initial states, end-states, and residual optionality in L2 acquisition. In: Proceedings of the 23rd Boston University Conference on Language Development. Somerville, MA: Cascadilla Press, 1999.

. Native language attrition and developmental instability at the syntax-discourse interface: Data, interpretations and methods. Bilingualism: Language and Cognition, 7, 2004:143-145.

THORNTON, A. Morfologia. Roma: Carocci, 2005.

THORNTON, A.; IACOBINI, C. Tendenze nella formazione delle parole nell'italiano del ventesimo secolo. In: MORETTI, B.; PETRINI, D.; BIANCONI, S. (Orgs.) Linee di tendenza dell'italiano contemporaneo. Roma: Bulzoni, 1992:22-55.

Fonti e stratificazione diacronica del lessico di base italiano. In: NAVARRO SALAZAR, M. T. (Org.) Italica Matritensia. Firenze: Cesati, 1997: 493-509. 
THORNTON, A.; IACOBINI, C.; BURANI, C. BDVDB. Una base di dati sul vocabolario di base della lingua italiana. Roma: Bulzoni, 1997.

TSIMPLI, I. et al. Subject in Ll attrition: evidence from Greek and Italian Near-Native speakers of English. In: Proceedings of the 26th Boston University Conference on Language Development, Sommerville MA, Cascadilla Press, 2002.

VANVOLSEM, S.; VERMANDERE, D.; MUSARRA, F; D'HULST, Y. (Orgs.) L'italiano oltre frontiera. Atti del $V^{\circ}$ Convegno Internazionale del Centro di Studi Italiani (Lovanio, 22-25 aprile 1998). Firenze-Lovanio: Franco Cesati Editore; Leuven: Leuven University Press, 2000, 2 voll. 